\title{
„Ohne Zusammenarbeit würde das gar nicht gehen“" Kokonstruktive Lehrpersonen-Kooperation bei der Unterrichtsentwicklung zu personalisiertem Lernen
}

\author{
Rita Stebler (D) - Marco Galle $\cdot$ Christine Pauli $\cdot$ Kurt Reusser
}

Eingegangen: 27. Dezember 2020 / Überarbeitet: 30. Juni 2021 / Angenommen: 2. Juli 2021 / Online publiziert: 24. Juli 2021

(C) Der/die Autor(en) 2021

Zusammenfassung In der Studie wird untersucht, inwiefern sich die kokonstruktive Lehrpersonen-Kooperation in Schulen, die ihren Unterricht bottom-up in Richtung einer stärkeren Personalisierung des Lernens weiterentwickeln, verändert. Die Daten wurden im Zeitraum von drei Schuljahren in 43 Schulen mittels Fragebögen $(\mathrm{t} 1, \mathrm{t} 3)$ und in elf dieser Schulen zusätzlich durch Leitfadeninterviews (t1, t2, t3) erhoben. Wie die statistischen und inhaltsanalytischen Auswertungen übereinstimmend zeigen, kooperieren die Lehrpersonen bei der Unterrichtsentwicklung rege, mit unterschiedlichen Partner/innen und kokonstruktiv. Die kokonstruktive Kooperation nimmt im Untersuchungszeitraum bei der Planung, Entwicklung und Auswertung von Unterricht für personalisiertes Lernen sowie im Umgang mit unterrichtlichen und schulischen Herausforderungen deutlich zu. Es entstehen Kooperationskulturen, die nicht nur die Unterrichtsentwicklung voranbringen, sondern auch bei beruflichen und persönlichen Problemen starken Rückhalt geben. Die Ergebnisse zeigen, dass unterrichtsbezogene Innovationen, die von den einzelnen Schulen ausgehen und gemeinsam bejahte Ziele verfolgen, die kokonstruktive Kooperation zwischen Lehrpersonen fördern und zum pädagogischen Handeln als kollegiale Lerngemeinschaften beitragen.

Dr. phil. Rita Stebler $(\bowtie) \cdot$ Prof. em. Dr. Kurt Reusser

Institut für Erziehungswissenschaft, Universität Zürich, Freiestrasse 36, 8032 Zürich, Schweiz

E-Mail: stebler@ife.uzh.ch

Prof. em. Dr. Kurt Reusser

E-Mail: reusser@ife.uzh.ch

Dr. phil. Marco Galle

Abteilung Ausbildung, Pädagogische Hochschule Zürich, Lagerstrasse 2, 8090 Zürich, Schweiz

E-Mail: marco.galle@phzh.ch

Prof. Dr. Christine Pauli

Departement Erziehungs- und Bildungswissenschaften, Zentrum für Lehrerinnen- und Lehrerbildung, Universität Freiburg/Fribourg, Rue Faucigny 2, 1700 Fribourg, Schweiz

E-Mail: christine.pauli@unifr.ch 
Schlüsselwörter Lehrerkooperation · Kollegiale Lerngemeinschaft ·

Kokonstruktion · Personalisiertes Lernen · Mixed-Methods-Studie

\title{
It simply wouldn't work without cooperation: Co-constructive teacher cooperation in the development of personalized learning
}

\begin{abstract}
This study examines changes in co-constructive teacher collaboration in schools that develop their teaching bottom-up, increasingly personalizing learning. Data were collected using questionnaires $(\mathrm{t} 1, \mathrm{t} 3)$ from 43 schools over three school years. Additional guided interviews were conducted in eleven of these schools ( $\mathrm{t} 1, \mathrm{t} 2$, t3). The statistical and content-analytical evaluations consistently show that teachers frequently cooperate co-constructively with one another when developing personalized learning environments. Co-constructive collaboration increases significantly when teachers are planning, developing, and evaluating lessons and when they are dealing with teaching and academic challenges. Cultures of collaboration emerge that not only advance instructional development but also provide strong support for professional and personal problems. The results show that instructional innovations that originate in single schools, but share mutually agreed upon learning goals, promote co-constructive cooperation among teachers and contribute to creating supportive and collegial pedagogical communities.
\end{abstract}

Keywords Teacher cooperation · Community of Practice $\cdot$ Co-construction · Personalized Learning · Mixed Methods Study

\section{Einleitung}

Die Entwicklung heterogenitätssensibler Lehr-Lernumgebungen hat gegenwärtig - auch in der Schweiz - hohe pädagogische Priorität (Reusser et al. 2013). Spätestens seit der Einführung der integrativen Schulform legen die Bildungsinstitutionen großen Wert auf einen individuell lernförderlichen Unterricht und eine Vielfalt bejahende Schulkultur. Zahlreiche Volksschulen entwickeln teils variantenreiche Unterrichtsarchitekturen für personalisiertes Lernen (Stebler et al. 2018). Sie legen (Jahrgangs-) Klassen zu alters- und/oder leistungsdurchmischten Stammklassen zusammen, flexibilisieren die Lernzeiten, orchestrieren klassische und innovative LehrLernformen, kombinieren geführten und offenen Unterricht, gewähren den Lernenden Autonomiespielräume und praktizieren vielfältige Formen des individualisierenden und kooperativen Lernens sowie der individuellen Lernunterstützung. So schaffen sie Rahmenbedingungen und Strukturen für Unterricht, der kompetenzorientiertes und selbstgesteuertes Lernen unter konsequenter Berücksichtigung personaler Lern- und Bildungsvoraussetzungen ermöglichen soll (Stebler et al. 2021b). Die Entwicklungen werden von den Kollegien der einzelnen Schulen - autonom oder angestoßen durch äußere Umstände - initiiert und in jahrelanger Arbeit schrittweise gemeinsam umgesetzt (Galle im Druck). Auch unter der Zielsetzung einer verstärkten Personalisierung des Lernens ist eine erfolgreiche Unterrichtsentwicklung von der Lehrpersonen-Kooperation abhängig (Gross et al. 2018). Bisher weiß man jedoch 
wenig über Prozessqualitäten der Kooperation zwischen Lehrpersonen, die bottomup Lehr-Lernumgebungen entwickeln, um Schüler*innen heterogener Lerngruppen stärker im Einklang mit ihren personalen Lernvoraussetzungen zu fördern. Im vorliegenden Beitrag wird bezogen auf einen Zeitraum von drei Schuljahren folgender Frage nachgegangen: Inwiefern verändert sich die kokonstruktive LehrpersonenKooperation in Schulen, die ihren Unterricht mit Blick auf eine stärkere Personalisierung des Lernens weiterentwickeln? Nach der Situierung der Fragestellung im Forschungskontext (Kap. 2) wird die längsschnittlich angelegte Mixed-MethodsStudie zur Lehrpersonen-Kooperation beschrieben (Kap. 3). Anschließend werden Ergebnisse zu Häufigkeiten, Gegenstandsbereichen und Veränderungen der kokonstruktiven Lehrpersonen-Kooperation berichtet (Kap. 4) und diskutiert (Kap. 5).

\section{Kokonstruktive Lehrpersonen-Kooperation bei der Unterrichtsentwicklung zu personalisiertem Lernen}

Bildungsinstitutionen stehen heute stärker denn je unter Druck, alle Schüler/innen in heterogenen Lerngruppen so zu fördern, dass sie ihre Potenziale optimal entfalten können und auf lebenslanges Lernen vorbereitet werden. Um dieser Forderung in Anbetracht von vielfältiger Schülerschaft, Digitalisierung und Kompetenzorientierung nachzukommen, setzen Schulen insbesondere im englischsprachigen Bildungsraum zunehmend auf ,,personalized learning“ (Murphy et al. 2016; Zhang et al. 2020). Personalisiertes Lernen ist kein klar umrissenes pädagogisches Konzept, sondern ein Sammelbegriff, der bekannte (reform-) didaktische Ansätze (z. B. offener Unterricht, Differenzierung) weiterentwickelt und das Lernen mit digitalen Medien einschließt (Lee et al. 2021; Schaumburg 2021; Stebler et al. 2021a, b). Auch in der Schweiz arbeiten die Lehrpersonen vieler Volksschulen seit langem gemeinsam und mit Erfolg daran, die ursprünglich frontalunterrichtlich geprägte, didaktische Tradition durch Lehr-Lernszenarien zu ergänzen, mit denen eine stärkere Förderung des selbstgesteuerten, eigenverantwortlichen und dialogisch-kooperativen Lernens angestrebt wird. Aus pädagogisch-psychologischer Sicht lassen sich im internationalen Personalisierungsdiskurs fünf Tiefendimensionen feststellen, die in vielfältiger Konfiguration in zahlreichen Bildungskontexten als Ziele und Handlungsorientierungen einer personalisierten Lernkultur vorkommen (Stebler et al. 2018): (1) Unterrichtsangebote an die personalen Bildungs- und Lernvoraussetzungen von Lernenden und Lerngruppen anpassen, (2) personale und soziale Kompetenzen aufbauen sowie Schüler/innen in ihrer Persönlichkeit fördern, (3) selbstgesteuertes Lernen auf eigenen Wegen ermöglichen, (4) kompetenzorientiertes Lernen zur persönlichen Sache machen und (5) als Lehrperson und Lerngemeinschaft bildend und unterstützend wirken. Die Schüler/innen werden dabei verstärkt als Subjekte von Bildungsprozessen wahrgenommen, die bei der Angebotsgestaltung fassbar mitwirken und zunehmend Verantwortung für das eigene Lernen übernehmen sollen (Schratz et al. 2011).

Schulen, welche die hergebrachte ,grammar of schooling“ (Tyack und Tobin 1994) aufbrechen und personalisiertes Lernen ermöglichen wollen, müssen stark in Unterrichtsentwicklung investieren (Haenisch und Steffens 2017). Zu den vorrangigen, auf die Optimierung qualitätsvoller fachlicher und überfachlicher Lernprozes- 
se bezogenen Entwicklungsaufgaben zählen: ein schulspezifisches konsensfähiges Personalisierungskonzept ausarbeiten, entsprechende Lehr-Lernszenarien gestalten, individuell anschlussfähige Lernangebote bereitstellen, die Schüler/innen für selbstgesteuertes Lernen qualifizieren und die individuelle Lernunterstützung verstärken (Reusser 2019; Stebler et al. 2018). Um in den einzelnen Schulen so weitreichende Veränderungen der Lernkulturen herbeizuführen und zum ,courant normal“ werden zu lassen, ist die intensive Kooperation zwischen Lehrpersonen (Grosche et al. 2020) eine „nicht hintergehbare Voraussetzung“ (Bastian 2007, S. 207).

„Kooperation ist gekennzeichnet durch den Bezug auf andere, auf gemeinsam zu erreichende Ziele bzw. Aufgaben, sie ist intentional, kommunikativ und bedarf des Vertrauens. Sie setzt eine gewisse Autonomie voraus und ist der Norm der Reziprozität verpflichtet“ (Spieß 2004, S. 199). Kooperation zwischen Lehrpersonen ist, wenn man sich an diese in der Organisationspsychologie beheimatete und im deutschsprachigen Bildungsraum breit rezipierte Definition hält, ein komplexes Phänomen, das in verschiedenen Theorie- und Forschungszusammenhängen untersucht wird (Fussangel und Gräsel 2014; Rolff 2015; Vangrieken et al. 2017). Mit Bezug auf Ansätze des situierten Lernens können Lehrpersonen, die aus eigener Initiative bzw. bottom-up gemeinsam Unterricht für personalisiertes Lernen entwickeln, als „Communities of Practice“ (CoP) (Wenger 1998) betrachtet werden. CoP sind selbstorganisierte Teams, deren Mitglieder vor ähnlichen Herausforderungen stehen und innerhalb ihrer Institution kooperieren, um gemeinsame Standards und Normen zu schaffen, berufspraktische Probleme zu lösen, Innovationen voranzutreiben und voneinander zu lernen. Sie verfügen über kollektive Wissensbestände, die sich auf Grund einer gemeinsamen Praxis, eines gemeinsamen Diskurses und gemeinsamer Artefakte ergeben (Bloh und Bloh 2016). Kooperation in CoP hilft, die Grenzen der individuellen Leistungsfähigkeit zu überwinden und kann unterschiedliche Formen und Prozessqualitäten annehmen.

Gräsel et al. (2006) unterscheiden in ihrem mit Chemielehrpersonen entwickelten Modell drei Formen der unterrichtsbezogenen Lehrpersonen-Zusammenarbeit, nämlich Austausch, arbeitsteilige Kooperation und - als anspruchsvollste und intensivste Form - kokonstruktive Kooperation. Sie gehen davon aus, ,dass diese verschiedenen Kooperationsformen unterschiedliche Funktionen im Rahmen der Lehrerarbeit haben und dass sie ihren Zweck je nach zu bearbeitender Aufgabe erfüllen“" (Fussangel und Gräsel 2014, S. 851). Bei der Unterrichtsentwicklung spielt die kokonstruktive Kooperation eine entscheidende Rolle (Harazd und Drossel 2011). Diese

- beinhaltet, gemeinsame, zyklische, intensive und interdependente Aushandlungen und Reflexionen von Innovationen und ihren Konkretisierungen" (Grosche et al. 2020, S. 467);

- erfolgt, wenn ganze Schulen beteiligt sind, in unterschiedlichen, sich teils überlappenden und inspirierenden Aktivitätskreisen (u. a. Pädagogisches Team, Fachschaft, Schulkonferenz) (Haenisch und Steffens 2017);

- manifestiert sich u.a. (1) als gemeinsame Planung, Entwicklung und Auswertung von Unterricht, (2) als Diskussion, Konsensfindung und Unterstützung bei unterrichts- und schulrelevanten Herausforderungen sowie (3) als gemeinsam verantwortete Unterrichtsdurchführung (Keller-Schneider und Albisser 2013). 
Dadurch, dass CoP Prozesse und Wirkungen der kokonstruktiven Kooperation gewohnheitsmäßig reflektieren und strukturelle (z.B. überlappende Arbeitszeiten), schulkulturelle (z. B. unterstützende Schulleitung) und individuelle (z. B. erlebte Autonomie) Rahmenbedingungen sowie soziale Vorbedingungen (z. B. Zielinterdependenz, Bereitschaft zu Deprivatisierung von Unterricht) laufend optimieren, steigern sie die Erträge und die Prozessqualität der gemeinsamen Unterrichtsentwicklung (Bonsen und Rolff 2006; Grosche et al. 2020; Rolff 2015).

Kokonstruktive Lehrpersonen-Kooperation verändert sich im Zuge der Unterrichtsentwicklung, ist anspruchsvoll und besonders anfänglich gewöhnungsbedürftig. Sie tangiert vertraute Arbeitsbeziehungen, geläufige Praktiken sowie etablierte Normen der Berufskultur (u.a. Kollegialität als Nichteinmischung) und schränkt den individuellen Gestaltungsspielraum ein. In frühen Phasen der gemeinsamen Unterrichtsentwicklung sind Terminfindungsprobleme, verlängerte Arbeitszeiten, Unsicherheiten, Vorbehalte gegenüber Kooperationspartner/innen und Konflikte unausweichlich (Muckenthaler et al. 2019). Damit Lehrpersonen sich trotzdem auf intensive Kooperation einlassen, muss sie ihnen notwendig und sinnvoll erscheinen (Pröbstel und Soltau 2012). Förderlich sind zudem ähnliche didaktische Präferenzen, günstige Rahmenbedingungen und eine unterstützende Schulleitung (Harazd und Drossel 2011; Rolff 2015; Trumpa et al. 2016). Beim Zusammenarbeiten lernen die Teammitglieder einander und sich selbst besser kennen, wodurch Sicherheit, Akzeptanz und Wertschätzung zunehmen (Kuper und Kapelle 2012). Infolge gemeinsamer Zielbindung, Aufgabenorientierung und komplexer Aushandlungsprozesse werden ihre beruflichen Beziehungen allmählich enger. Die Teammitglieder generieren kollektive Wissensbestände, erweitern und vertiefen die eigenen professionellen Kompetenzen, werden offener für Neues und erleben höhere Selbstwirksamkeit. Unterstützung und Rückhalt im Kollegium erzeugen positive Gefühle, steigern das berufliche Wohlbefinden und entlasten bei Schwierigkeiten (Gast et al. 2017).

Kooperation gehört zum Berufsauftrag von Lehrpersonen und ist ein Qualitätsmerkmal guter Schulen. Dennoch werden vor allem elaborierte Kooperationsformen im deutschsprachigen Bildungsraum erst zögerlich realisiert (Massenkeil und Rothland 2016; Trumpa et al. 2016). Im Unterrichtsalltag erfolgt LehrpersonenKooperation selten in Formen, die über gegenseitige Hilfeleistungen und einen raschen, anlassbezogenen Austausch von Informationen und Materialien hinausgehen (Richter und Pant 2016; Soltau und Mienert 2009), beschränkt sich häufig auf organisatorische Subeinheiten der Schule, tritt vorwiegend innerhalb von Fach- und Jahrgangsgrenzen auf, nimmt mit steigender Schulstufe ab (Halbheer et al. 2008; Kuper und Kapelle 2012; Steinert et al. 2006) und bezieht sich am ehesten auf sonderpädagogische Maßnahmen (Lütje-Klose und Miller 2017) sowie auf Unterrichtsplanung und -reflexion (Halbheer et al. 2008). Unterrichtet wird üblicherweise allein (Kuper und Kapelle 2012). Vergleichsweise häufiger und teils in intensiven Formen kooperieren die Lehrpersonen bei der Implementation unterrichtlicher oder schulischer Innovationen (Bennemann und Schönknecht 2016; Hartmann et al. 2020; OECD 2014; Reusser et al. 2013). Dies zeigen auch Begleitstudien zur Ermöglichung personalisierten Lernens aus dem englischsprachigen Bildungsraum (Basham et al. 2016; Pane et al. 2017; Prain et al. 2015; Sebba et al. 2007). Bisher weiß 
man aber wenig über Ausprägungen und Veränderungen der kokonstruktiven Lehrpersonen-Kooperation bei der Unterrichtsentwicklung zu personalisiertem Lernen. Vor diesem Hintergrund wird in der nachfolgend beschriebenen Studie mit Blick auf einen Zeitraum von drei Schuljahren untersucht, inwiefern und bei welchen Gegenständen sich die kokonstruktive Kooperation zwischen Lehrpersonen verändert, wenn sie den Unterricht in ihrer Schule bottom-up in Richtung einer stärkeren Personalisierung des Lernens weiterentwickeln.

\section{Methode}

Die Untersuchung zur Lehrpersonen-Kooperation ist Teil der perLen-Studie (personalisierte Lernkonzepte in heterogenen Lerngruppen ${ }^{1}$ ) in der 65 Schulen, die bei der Entwicklung heterogenitätssensibler Lehr-Lernumgebungen Pionierarbeit leisten, im Längsschnitt (Schuljahre 2012/13-2014/15) untersucht wurden. Die mehrheitlich öffentlichen Schulen mit Primar- und/oder Sekundarstufe I, deren Unterricht im Hinblick auf eine stärkere Personalisierung des Lernens deutlich von traditionellen unterrichtsgrammatischen Formen abweicht (Stebler et al. 2021b), haben sich für die Studienteilnahme beworben oder wurden eingeladen. Jede Schule der Studie (=perLen-Schule) hat ein eigenes Signalement, ein schulspezifisches Personalisierungskonzept, welches den lokalen und personellen Voraussetzungen und Gegebenheiten sowie den pädagogischen Werten des Kollegiums Rechnung trägt und ausgewählte Personalisierungskriterien (z.B. selbstgesteuertes Lernen ermöglichen) in den Vordergrund rückt (Galle im Druck), und steht bei der Unterrichtsentwicklung an einem anderen Ort. Alle Schulen haben mindestens ein Jahr vor der ersten Datenerhebung mit Unterrichtsentwicklung zu personalisiertem Lernen begonnen. Beispiele für Entwicklungsprojekte, welche die Lehrpersonen als Arbeits-, Lern- und Qualitätsteams (Brägger et al. 2021) im Untersuchungszeitraum u. a. vorangetrieben haben, sind:

- [Schule L] das Ausarbeiten von Kompetenzrastern und darauf bezogener kognitiv aktivierender, individuell anschlussfähiger Lernaufgaben für personalisierte Lernangebote in der Sekundarstufe I. Die Aufgaben wurden von den Fachschaften erarbeitet, durch wiederholte Vernehmlassungen in den Lernlandschaftsteams verbessert, fachübergreifend koordiniert, zu Themendossiers gebündelt und samt Unterrichtsskizzen allen Lehrpersonen elektronisch zugänglich gemacht.

- [Schule E] die Weiterentwicklung des Coachingsystems. Unter der Moderation einer für Lerncoaching zertifizierten Lehrperson der Schule E wurden die klassenspezifischen Coachingpraktiken gemeinsam reflektiert, mit Blick auf Inhalte, Gesprächsführung und Dokumentation im zyklischen Zusammenspiel zwischen Projektteam, Lernlandschaftsteams und Konvent schulweit vereinheitlicht und schrittweise so umgesetzt, dass jede/r Schüler/in während der Verweildauer in der Sekundarstufe I alle drei Wochen mit jeweils derselben Lehrperson ein standardisiertes Coachinggespräch hat.

\footnotetext{
1 Die Studie wurde gefördert von der Stiftung Mercator Schweiz.
} 
- [Schule A] die Weiterentwicklung des Lerntagebuchs durch die Stufenteams in periodischer Rücksprache mit dem Kollegium von einem Dokumentations- zu einem Planungs- und Reflexionsinstrument, auf dessen Grundlage jede jahrgangsdurchmischte vierköpfige Sitz- bzw. Lerngruppe einmal wöchentlich gemeinsam Arbeitsrückschau hält.

Pro Schuljahr (t1, t2, t3) wurde in jeder perLen-Schule eine Online-Befragung der Lehrpersonen (geschlossene Fragen; Klassenstufen 4-9) durchgeführt. Zusätzlich wurden in elf dieser perLen-Schulen (=perLen-Fallschulen) die Schulleitungen (t1) und dreimal ( $\mathrm{t} 1, \mathrm{t} 2, \mathrm{t} 3)$ dieselbe Lehrpersonengruppe interviewt (Leitfadeninterviews).

In den Online-Befragungen mussten die Lehrpersonen angeben, wie häufig (fast jeden Tag $=5$, weniger als einmal pro Monat $=1$ ) sie in ihrer Schule mit bestimmten Personengruppen zusammenarbeiten (t1: 5 Items) und wie oft $(\mathrm{sehr}$ oft $=5$, nie $=1)$ sich die Zusammenarbeit auf ausgewählte Gegenstände der Unterrichtsentwicklung zu personalisiertem Lernen bezieht (t1, t3: 17 Items; u. a. Entwicklung von Unterrichtsmaterialien). Ausgewertet (SPSS Version 25; Frequenz- und Unterschiedsanalysen) wurden die Fragebogendaten jener 178 Lehrpersonen, die zweimal (t1, t3) Kooperationsitems beurteilt haben. Diese Lehrpersonen (Frauen: 62\%) stammen aus 43 perLen-Schulen (Gesamtstichprobe), unterrichten vorwiegend auf der Sekundarstufe I (76\%), sind durchschnittlich 44 Jahre alt ( $\mathrm{SD}=11$ Jahre) und haben im Mittel 17 Jahre ( $\mathrm{SD}=11$ Jahre) Unterrichtserfahrung, davon rund 10 Jahre (SD=8 Jahre) in ihrer perLen-Schule. Sie unterrichten die curricularen Fächer (85\%) oder erteilen Spezialunterricht (15\%; z. B. Schulische Heilpädagogik).

Bei den halbstrukturierten leiffadengestützten Interviews in den perLen-Fallschulen (11/43) wurden u.a. Praxis und Entwicklung der Lehrpersonen-Kooperation thematisiert. Die Leitfrage bei t2 bspw. lautete: „Lehrpersonen sind je länger desto weniger Solo-Player. Sie bilden Teams - pädagogische Teams, Stufenteams, Schulhausteams usw. - und kooperieren auf vielfältige Weise. Könnten Sie uns etwas zur Kooperationspraxis und zur Teamentwicklung im laufenden Schuljahr sagen?" Die insgesamt 45 Interviews wurden aufgezeichnet, wörtlich transkribiert und mit Hilfe des Analyseprogramms MAXQDA und einer inhaltlich strukturierenden Inhaltsanalyse (Kuckartz 2018) in drei Schritten codiert: Zuerst wurde das gesamte Datenmaterial (ca. 630.000 Wörter) anhand einer Basiscodierung mit je einer Basiskategorie für die fünf Schulentwicklungsbereiche (1) Unterrichtsentwicklung, (2) Entwicklung organisationaler Strukturen, (3) Personalentwicklung, (4) Entwicklung des Schullebens und (5) Entwicklung der Kooperation zwischen Lehrpersonen vorstrukturiert. Eine erste Qualitätskontrolle, bei der zwei Personen unabhängig voneinander dasselbe Interview codierten, ergab eine $81 \%$ ige Übereinstimmung (Cohens Kappa =0,77). Bei den Abweichungen einigten sich die Codierenden auf eine einheitliche Zuordnung der betreffenden Fundstellen (Schmidt 2013). Danach erfolgte die Feincodierung aller Fundstellen (Textstellen $\geq$ fünf Wörter) zur Entwicklung der Lehrpersonen-Kooperation (ca. 50.000 Wörter). Das Kategoriensystem wurde in Anlehnung an die Kooperationstypologien von Gräsel et al. (2006) sowie von Keller-Schneider und Albisser (2013) entwickelt und induktiv justiert. Es resultierten die drei Hauptka- 
tegorien: Austausch (1), Arbeitsteilung (2) und Kokonstruktion (3). Kokonstruktion wurde zudem in drei Subkategorien ausdifferenziert:

- (3a) Gemeinsame Planung, Entwicklung und/oder Auswertung von Unterricht im Hinblick auf eine stärkere Personalisierung des Lernens (u. a. Unterrichtsthemen festlegen, Lernziele formulieren, Aufgabensammlungen erstellen, Stoff- und Unterrichtspläne entwickeln).

- (3b) Diskussion, Konsensfindung und Unterstützung bei unterrichts- und schulrelevanten Herausforderungen (u. a. diskursiv einen Konsens bzgl. Lehr-Lernformen, Lernzielen und Lernbegleitung erarbeiten; eine gemeinsam geteilte Kooperationskultur im Kollegium aufbauen; einander bei beruflichen Problemen und persönlichen Krisen unterstützen; periodisch das Lern- und Leistungsverhalten der einzelnen Schüler/innen besprechen).

- (3c) Gemeinsam verantworteter Unterricht (u. a. Unterricht gemeinsam durchführen; schülerbezogene Leistungserwartungen abgleichen; professionelle Ressourcen zwecks adaptiver Lernunterstützung koordinieren).

Eine zweite verfahrensgleiche Qualitätskontrolle ergab für die Feincodierung einen Wert von $91 \%$ (Cohens Kappa=0,89).

\section{Ergebnisse}

Bei der Unterrichtsentwicklung zu einer stärkeren Personalisierung des Lernens kooperieren die Lehrpersonen der untersuchten Schulen sowohl spontan und informell als auch in institutionalisierten, sich teils überlappenden schulspezifischen Aktivitätskreisen (u.a. pädagogisches Team, Stufenteam, Lernlandschaftsteam, Entwicklerteam, Coaching-Team, Fachschaft) (Galle im Druck). Die Kooperation ist rege und auch kokonstruktiv (4.1). Die kokonstruktive Lehrpersonen-Kooperation nimmt im Untersuchungszeitraum von drei Schuljahren deutlich zu (4.2) und wird zudem intensiver (4.3).

\subsection{Die Lehrpersonen kooperieren rege und kokonstruktiv}

Wie die Ergebnisse für die Teilstichprobe der perLen-Fallschulen (FS) und für die Gesamtstichprobe der perLen-Schulen (S) übereinstimmend zeigen, kooperieren die untersuchten Lehrpersonen rege. Zu Beginn des dreijährigen Untersuchungszeitraums (t1) kooperierte rund die Hälfte von ihnen (fast) täglich mit Regellehrpersonen (FS: 56\%; S: 51\%), mehrmals pro Woche oder häufiger mit Personen der Schulleitung (FS: 56\%; S: 53,9\%), mit Teamteaching-Partner/innen (FS: 49\%; S: $47 \%$ ) und/oder mit schulischen Heilpädagog/innen (FS: $45 \%$; S: $51 \%$ ) sowie mindestens einmal pro zwei Monate mit weiteren Speziallehrpersonen (u.a. Deutsch als Zeitsprache; FS: 52\%; S: 52\%). In den Interviews äusserten sich die Lehrpersonen vorwiegend über kokonstruktive Kooperation. Bei der Basiscodierung der 45 transkribierten Interviews mit Schulleitungen und Lehrpersonengruppen der Fallschulen wurden 399 Fundstellen (F) der Kategorie ,Entwicklung der Kooperation zwischen Lehrpersonen` zugeordnet. Der Feincodierung dieser Fundstellen zufol- 
ge berichteten Schulleitungen und Lehrpersonen am häufigsten über kokonstruktive Kooperation $(\mathrm{F}=267 ; 67 \%)$, gefolgt von Austausch $(\mathrm{F}=73 ; 18 \%)$ und Arbeitsteilung $(\mathrm{F}=59 ; 15 \%)$. Bei der Codierung der Fundstellen zur kokonstruktiven Kooperation nach Gegenstandsbereichen zeigte sich, dass mehrheitlich die gemeinsame ,Planung, Entwicklung und/oder Auswertung von Unterricht" thematisiert wurde $(\mathrm{F}=143 ; 54 \%)$. Aussagen $\mathrm{zu}$,Diskussion, Konsensfindung und Unterstützung bei unterrichts- und schulrelevanten Herausforderungen' kamen ebenfalls häufig vor $(\mathrm{F}=99 ; 37 \%)$. Deutlich seltener wurde über kokonstruktive Kooperation mit Blick auf ,gemeinsam verantworteten Unterricht' gesprochen $(\mathrm{F}=25 ; 9 \%)$.

\subsection{Die kokonstruktive Lehrpersonen-Kooperation nimmt im Untersuchungszeitraum zu}

Die Lehrpersonen gaben zu Beginn (t1) und gegen Ende (t3) des dreijährigen Untersuchungszeitraums anhand derselben Items an, wie häufig sich ihre Zusammenarbeit auf ausgewählte Gegenstände bzw. Aufgaben bei der Unterrichtsentwicklung zu personalisiertem Lernen bezieht. In Tab. 1 sind die Ergebnisse (t1, t3) für die Fallschulen (FS) und die Gesamtstichprobe (S) dargestellt. Die Gruppierung der Items folgt den Kategorien der Inhaltsanalyse.

Wenn man die Häufigkeiten nach Kategorien betrachtet, zeigt sich folgendes Bild: Die Lehrpersonen (1) tauschen häufig Unterrichtserfahrungen aus, sprechen Termine $\mathrm{ab}$ und geben Unterrichtsmaterialien weiter. Kokonstruktive Kooperation kommt gelegentlich vor. Die Lehrpersonen

- (3a) geben manchmal neue pädagogische Ideen weiter, erproben neue didaktische Ansätze und entwickeln gemeinsam Unterrichtsmaterialien.

- (3b) kooperieren manchmal bei der individuellen Förderung der Schüler/innen, indem sie gemeinsam Schülerleistungen beurteilen, Lern- und Förderziele formulieren, Lernstände diagnostizieren, Förderpläne erstellen sowie individuelle Lernprogramme oder Lernkonzepte entwickeln. Zudem kooperieren sie bei Problemen mit der Klasse oder der eigenen Arbeit.

- (3c) bereiten den Unterricht zwar manchmal gemeinsam vor, unterrichten aber eher selten im Teamteaching. Es kommt nur gelegentlich vor, dass sie gemeinsam Unterricht reflektieren oder Hausaufgaben abstimmen.

Im Längsschnitt (t1- $\mathrm{t} 3$ ) betrachtet, zeigt sich bei den meisten Kooperationsgegenständen ein signifikanter, teils mittel bis stark ausgeprägter Frequenzanstieg (Tab. 1): Bei der Kooperationsform Austausch (1) haben im Untersuchungszeitraum die Terminabsprachen sowie der Austausch von Unterrichtsmaterialien deutlich zugenommen. Auch bei der kokonstruktiven Kooperation sind die Frequenzen, ausser bei der Kategorie „Gemeinsam verantworteter Unterricht“, stark gestiegen.

- (3a) Mit Blick auf die Kategorie „Gemeinsame Planung, Entwicklung und Auswertung von Unterricht“" zeigt sich, dass die Lehrpersonen gegen Ende des Untersuchungszeitraums vergleichsweise häufiger neue pädagogische Ideen weitergeben, gemeinsam neue Unterrichtsideen und Methode erproben sowie Unterrichtsmaterialien entwickeln. 
Tab. 1 Häufigkeit der Zusammenarbeit von Lehrpersonen bei der ersten und dritten Online-Befragung im Rahmen der perLen-Studie

\begin{tabular}{|c|c|c|c|c|c|c|c|c|c|c|c|c|c|c|}
\hline \multirow[b]{2}{*}{$\begin{array}{l}\text { Wie oft bezieht sich die } \\
\text { Zusammenarbeit auf } \\
\text { folgende Gegenstände? }\end{array}$} & \multicolumn{7}{|c|}{$\begin{array}{l}\text { perLen-Fallschulen (=Teilstichprobe: } 11 \text { Schulen } \\
\text { mit } 66 \text { Lehrpersonen) }\end{array}$} & \multicolumn{7}{|c|}{$\begin{array}{l}\text { perLen-Schulen (= Gesamtstichprobe: } 43 \text { Schulen mit } \\
178 \text { Lehrpersonen) }\end{array}$} \\
\hline & $\begin{array}{l}\mathrm{t} 1 \\
M\end{array}$ & $S D$ & $\begin{array}{l}\mathrm{t} 3 \\
M\end{array}$ & $S D$ & $t(65)$ & $p$ & $d$ & $\begin{array}{l}\mathrm{t} 1 \\
M\end{array}$ & $S D$ & $\begin{array}{l}\mathrm{t} 3 \\
\mathrm{M}\end{array}$ & $S D$ & $t(177)$ & $p$ & $d$ \\
\hline \multicolumn{15}{|l|}{ (1) Austausch } \\
\hline Terminabsprachen & 4,18 & 0,70 & 4,41 & 0,68 & $-2,16$ & 0,05 & 0,33 & 4,17 & 0,77 & 4,29 & 0,75 & $-1,69$ & n.s. & - \\
\hline $\begin{array}{l}\text { Austausch von Unter- } \\
\text { richtserfahrungen }\end{array}$ & 4,27 & 0,83 & 4,26 & 0,71 & 0,15 & n.s. & - & 4,22 & 0,81 & 4,14 & 0,80 & 1,22 & n.s. & - \\
\hline $\begin{array}{l}\text { Austausch von Unter- } \\
\text { richtsmaterialien }\end{array}$ & 3,91 & 1,02 & 4,35 & 0,83 & $-4,65$ & 0,001 & 0,47 & 3,79 & 1,05 & 4,06 & 0,93 & $-3,97$ & 0,001 & 0,27 \\
\hline \multicolumn{15}{|c|}{ (3a) Gemeinsame Planung, Entwicklung, Auswertung von Unterricht im Hinblick auf personal. Lernen } \\
\hline $\begin{array}{l}\text { Weitergabe neuer } \\
\text { pädagogischer Ideen }\end{array}$ & 3,00 & 0,89 & 3,64 & 0,82 & $-5,33$ & 0,001 & 0,78 & 3,01 & 0,92 & 3,47 & 0,90 & $-6,20$ & 0,001 & 0,51 \\
\hline $\begin{array}{l}\text { Erprobung neuer } \\
\text { Unterrichtsideen und } \\
\text { Methoden }\end{array}$ & 3,00 & 0,90 & 3,32 & 0,88 & $-2,83$ & 0,01 & 0,36 & 3,00 & 0,90 & 3,22 & 0,96 & $-2,76$ & 0,01 & 0,24 \\
\hline $\begin{array}{l}\text { Entwicklung von } \\
\text { Unterrichtsmaterialien }\end{array}$ & 3,25 & 1,04 & 3,83 & 1,06 & $-2,56$ & 0,05 & 0,55 & 3,35 & 1,27 & 3,60 & 1,09 & $-3,13$ & 0,01 & 0,21 \\
\hline $\begin{array}{l}\text { Beurteilung von } \\
\text { Schülerleistungen }\end{array}$ & 3,38 & 0,74 & 3,68 & 0,83 & $-2,41$ & 0,05 & 0,38 & 3,38 & 0,80 & 3,54 & 0,93 & $-2,13$ & 0,05 & 0,18 \\
\hline $\begin{array}{l}\text { Zielvorgaben für die } \\
\text { Schüler/innen }\end{array}$ & 3,38 & 0,78 & 3,76 & 0,95 & $-2,57$ & 0,05 & 0,44 & 3,31 & 0,88 & 3,57 & 0,97 & $-3,04$ & 0,01 & 0,28 \\
\hline $\begin{array}{l}\text { Förderplanung/ } \\
\text { Standortgespräche }\end{array}$ & 3,03 & 1,10 & 3,42 & 0,93 & $-3,34$ & 0,01 & 0,38 & 3,04 & 1,01 & 3,29 & 0,94 & $-3,60$ & 0,001 & 0,26 \\
\hline $\begin{array}{l}\text { Diagnose von Lern- } \\
\text { ständen }\end{array}$ & 2,97 & 1,04 & 3,26 & 0,95 & $-2,17$ & 0,05 & 0,29 & 2,96 & 0,97 & 3,17 & 0,89 & $-2,82$ & 0,01 & 0,23 \\
\hline $\begin{array}{l}\text { Individuelle Lernpro- } \\
\text { gramme/Lernkonzepte }\end{array}$ & 3,21 & 0,92 & 3,32 & 1,00 & $-0,88$ & n.s. & - & 3,12 & 1,04 & 3,26 & 1,01 & $-1,87$ & n.s. & - \\
\hline $\begin{array}{l}\text { Probleme mit der } \\
\text { Klasse }\end{array}$ & 3,33 & 0,88 & 3,83 & 0,89 & $-3,56$ & 0,001 & 0,57 & 3,34 & 0,96 & 3,89 & 0,86 & $-6,98$ & 0,001 & 0,61 \\
\hline $\begin{array}{l}\text { Persönliche Unzufrie- } \\
\text { denheit bzw. Probleme } \\
\text { mit der Arbeit }\end{array}$ & 2,97 & 1,01 & 3,5 & 0,90 & $-3,65$ & 0,01 & 0,56 & 2,80 & 1,03 & 3,51 & 0,93 & $-7,74$ & 0,001 & 0,72 \\
\hline (3c) Gemeinsam verantw & $\operatorname{er} U n$ & rricht & & & & & & & & & & & & \\
\hline $\begin{array}{l}\text { Gemeinsame Unter- } \\
\text { richtsvorbereitung }\end{array}$ & 3,12 & 1,09 & 3,36 & 1,08 & $-1,71$ & n.s. & - & 3,02 & 1,10 & 3,15 & 1,04 & $-1,55$ & n.s. & - \\
\hline $\begin{array}{l}\text { Gemeinsame Durch- } \\
\text { führung von Unterricht } \\
\text { (Teamteaching) }\end{array}$ & 2,64 & 1,29 & 2,65 & 1,17 & $-0,10$ & n.s. & - & 2,64 & 1,35 & 2,74 & 1,21 & $-1,11$ & n.s. & - \\
\hline $\begin{array}{l}\text { Gemeinsame Reflexi- } \\
\text { on und Nachbereitung } \\
\text { von Unterricht }\end{array}$ & 2,92 & 1,11 & 2,95 & 1,13 & 0,19 & n.s. & - & 2,84 & 1,13 & 2,83 & 1,08 & 0,13 & n.s. & - \\
\hline $\begin{array}{l}\text { Abstimmung des } \\
\text { Umgangs mit Haus- } \\
\text { aufgaben }\end{array}$ & 2,89 & 0,96 & 3,09 & 1,02 & $-1,25$ & n.s. & - & 2,80 & 1,09 & 2,94 & 1,10 & $-1,61$ & n.s. & - \\
\hline
\end{tabular}

Antwortformat: sehr oft (5), oft (4), manchmal (3), selten (2), nie (1)

$t 1$ und $t 3$ erste und dritte Befragung im Längsschnitt, $M$ Mittelwert, $S D$ Standardabweichung, $t(177)$ und $t(65)$ t-Wert und Freiheitsgrade, $p$ Signifikanzniveau, $d$ Effektstärke nach Cohen 
- (3b) Die Häufigkeit der kokonstruktiven Lehrpersonen-Kooperation hat auch bei der Diagnose von Lernständen, der Leistungsbeurteilung, der Förderplanung und den Zielvereinbarungen, nicht aber bei der Entwicklung individueller Lernprogramme deutlich zugenommen. Probleme mit der Klasse oder der eigenen Arbeit werden am Ende des Untersuchungszeitraums wesentlich häufiger gemeinsam angegangen.

- (3c) Keine Zunahme der kokonstruktiven Kooperation lässt sich beim Vorbereiten, Durchführen und Reflektieren von Unterricht sowie bei der Koordination von Hausaufgaben erkennen.

Wenn man die beiden Stichproben vergleicht, so sind zum einen die von den Lehrpersonen berichteten Kooperationshäufigkeiten in den perLen-Fallschulen und in der Gesamtstichprobe der perLen-Schulen sehr ähnlich. Dies gilt für beide Erhebungszeitpunkte. Zum anderen zeigt sich im Längsschnitt in der Teil- wie in der Gesamtstichprobe bei (fast) denselben Gegenständen ein deutlicher Anstieg der Kooperationsfrequenz.

\subsection{Prozessmerkmale und Veränderungen der kokonstruktiven Lehrpersonen- Kooperation}

Für die perLen-Fallschulen liegen sowohl Fragebogen- als auch Interviewdaten zu denselben Gegenständen und Kategorien der kokonstruktiven Lehrpersonen-Kooperation vor. Die Ergebnisse der strukturierenden qualitativen Inhaltsanalyse der Fundstellen, die den drei Subkategorien der kokonstruktiven Kooperation zugeordnet wurden, bestätigen die berichteten Frequenzsteigerungen. Zudem geben sie Auskunft über Prozessmerkmale und Veränderungen der kokonstruktiven Kooperation bei der Unterrichtsentwicklung zu personalisiertem Lernen. Nachstehend werden Ergebnisse der Inhaltsanalyse zu Prozessmerkmalen und Veränderungen in der Qualität der kokonstruktiven Kooperation unter Verwendung von Belegstellen aus den Interviews vertiefend beleuchtet. Die Quellenangaben erfolgen in der Form: Akteur $(\mathrm{SL}=$ Schulleitung, LP=Lehrperson), Schule $(\mathrm{A}-\mathrm{K})$, Erhebung (t1, t2, t3); z. B. LP_B_t1.

\subsubsection{Gemeinsame Planung, Entwicklung und Auswertung von Unterricht (3a)}

Bei der Entwicklung ihres Unterrichts folgt jede perLen-Schule einem schuleigenen Personalisierungskonzept, welches vom Kollegium erarbeitet, umgesetzt und laufend angepasst wird. ,[...] das kann ich als Einzelner nicht so realisieren [...]. Das braucht kollektive Anstrengungen“ (LP_H_t1). Wichtige Voraussetzungen für diesbezüglich intensive Kooperation sind anschließbare pädagogische Grundhaltungen, „offen sein wirklich für Neues und auch bereit sein, mal etwas zu machen oder etwas auszuprobieren“ (LP_H_t3) und eine reformwillige Schulleitung. Strategische Entscheidungen werden an periodischen Schulkonferenzen demokratisch getroffen und gemeinsam aufeinander abgestimmt. Dabei werden auch Zuständigkeiten geklärt, auseinanderlaufende Entwicklungstendenzen gebündelt sowie Kooperationsprozesse reflektiert. 
Die Personalisierungskonzepte sehen in der Regel vor, dass zwei bis drei Schulklassen zu größeren Lernverbänden, sogenannten Stammklassen, zusammengelegt werden, für die jeweils mehrere Lehrpersonen zuständig sind. ,[...] mit diesen Umstellungen kommt ein gewisser Druck. Auch [...] wegen den Lernaufträgen, die man machen muss, versuchen [wir] zusammenzuarbeiten“ (LP_H_t1). Vor allem anfangs ist dies anspruchsvoll, ,weil wir einfach auch noch relativ neu zusammen sind als Team, einfach, dass du wirklich jetzt mal gewisse Sachen ganz klar setzen musst, erarbeiten, zusammen erarbeiten“ (LP_E_t1). Da treffen verschiedene Meinungen aufeinander. „Bei uns ist das ein bisschen spannungsvoller, aber eben, wir sind auch die [größte Stammklasse der Schule], wir haben zweiundsechzig Schüler, wir sind fünf Lehrer [...], dann reden wir darüber, wie wir es umsetzen können, und rein das darüber sprechen wollen, löst Stress bei gewissen Leuten aus“"(LP_H_t3). Das Team muss sich finden. „Das heißt natürlich, man muss loslassen können“(LP_H_t2). Gegenseitige Wertschätzung, Lösungsorientierung, Kompromissfähigkeit und offene Kommunikation werden wiederholt als Voraussetzungen für kokonstruktive Kooperation genannt. „Alles was wir vorbereiten, das ist offen, alles was wir denken, auch [...] also, das ist für mich ein wichtiger Teaminhalt" (LP_D_t2). Wie aus den Interviews weiter hervorgeht, sinkt der Aushandlungsbedarf bei Grundsatzfragen jeweils rasch und auf der Basis gemeinsam geteilter grober Vorstellungen über Unterricht nach personalisierten Lernkonzepten konkretisieren sich bei der weiteren Zusammenarbeit Umsetzungsideen und Kooperationsmodalitäten.

Kokonstruktive Kooperation entsteht und entwickelt sich ferner durch gemeinsame Unterrichtsplanung, die häufig in fixen Zeitgefässen erfolgt. ,Wir [...] tun wirklich Unterrichtseinheiten zusammen erarbeiten, teilen es [...] auf oder machen [es] auch zusammen. [Die Qualität] ist sicher höher, als wenn wir es einzeln machen würden“ (LP_H_t2), nicht zuletzt, weil man sich auf Lernziele einigen muss. „Man hat nicht einfach irgendein Ziel und beginnt einmal mit Unterrichten“ (LP_H_t2). Manche Entwicklerteams geben Unterrichtspläne und Aufgabendossiers zur Vernehmlassung ins Kollegium. Sie ,machen alles fix fertig, [...] zeigen es den anderen, [arbeiten Rückmeldungen ein], und dann wird das für alle drei Zimmer kopiert, und wenn das Thema kommt, kannst du den Ordner nehmen und hast alles bereit [...]. Da bereiten nicht alle alles vor, sondern eben man unterstützt einander" (SL_J_t1). Die Zimmertüren stehen mehrheitlich offen und es gibt gegenseitige Unterrichtsbesuche mit Gelegenheiten für systematisches Beobachten. So wird bei der Unterrichtsentwicklung ein gemeinsam geteiltes Verständnis didaktischer Arrangements und auch klassenübergreifend eine hohe Umsetzungstreue personalisierter Lernkonzepte angestrebt.

\subsubsection{Diskussion, Konsensfindung und Unterstützung bei unterrichts- und schulrelevanten Herausforderungen ( $3 b)$}

Kokonstruktive Kooperation und diesbezügliche Veränderungen werden besonders häufig im Zusammenhang mit förderorientierter Leistungsbeurteilung als Voraussetzung für personalisierte Lernangebote und individuelle Lernunterstützung angesprochen. „Und auf Schulebene sind es mehr so Geschichten wie die Beurteilung. Also, wie gewährleisten wir, [...] dass es nicht darauf ankommt, in [welcher Stammklas- 
se] man war. [...] Das sind so Sachen, die wir über die letzten [...] Jahre definiert haben. Auch die Coachinggespräche: Da könnten Sie jetzt in den anderen [Stammklassen] fragen gehen. Das wäre identisch. Das haben wir generalisiert“ (LP_E_t1). Konkrete Beurteilungsaufgaben werden vermehrt als Team bewerkstelligt. „Was für mich noch äußerst wertvoll und auch noch ein wesentlicher Unterschied zu früher ist, dass ich nicht mehr Einzelkämpfer bin. Ich kann durchaus mal meine zwei anderen Coaches, die ebenfalls präsent sind, fragen, wie sie [bestimmte Lernende] wahrnehmen oder was sie beobachtet haben. [...] und wir können gemeinsam auch einen Schüler beurteilen. [...] Hier sind wir immer mehrere zusammen, und es gibt verschiedene Blickwinkel. Das ist sicher eine Stärke“ (LP_G_t1). Neben Beobachtungen und Gesprächen ermöglichen gesammelte Schülerdokumente (u. a. Lernjournale, Coachingprotokolle, Kompetenzraster) den Teammitgliedern, die Lern- und Leistungsentwicklung der einzelnen Schüler/innen gemeinsam zu verfolgen sowie Coaching- und Standortgespräche vorzubereiten. „Wir haben alle Lernwege sicher im Computer, auch die [gelösten] Lernschritte. Man kann sie dann dort bewerten und beurteilen. Schließlich sieht man, ah, der steht dort und dieser da“" (LP_K_t1). Die Lehrpersonen-Kooperation verändert sich auch dadurch, dass alle Schüler/innen regelmäßig im pädagogischen Team besprochen und gemeinsam Fördermaßnahmen antizipiert werden. Im Vergleich zu früher sind die Absprachen jetzt ,inhaltlich viel differenzierter“ (LP_E_t2), besonders bei Übertritten. „Wir übergeben uns auch die Schülerinnen und Schüler sehr, sehr genau. [...] In den pädagogischen Teams findet [...] eine sehr starke Zusammenarbeit statt“" (SL_H_t1). Mit personalisierten Pflicht- und Wahlangeboten wird gemeinsam versucht, den individuellen Lernbedürfnissen der Schüler/innen gerecht zu werden. Zudem gibt es Stütz-, Förder- und/ oder Vertiefungskurse sowie zusammen vereinbarte Individuallösungen. „Dass sie zum Beispiel zu mir ins Wahlfach Französisch kommen, aber nur zwei Kurse besuchen können und den dritten nicht, damit sie doch noch [...] eine Doppelstunde Geometrie besuchen können“ (LP_H_t3).

Die Lehrpersonen, die im dreijährigen Untersuchungszeitraum gemeinsam eine Stammklasse unterrichten, wachsen als Team zusammen. Damit steigen, wie sie wiederholt ausführen, Transparenz und gegenseitiges Vertrauen. „Wir besprechen alles. Wir besprechen schwierige Situationen, wir besprechen, wie unsere Grundhaltung ist, wir besprechen Herangehensweisen, Konsequenzen, querbeet" (LP_E_t3). Indem die Lehrpersonen vor allem in den offenen Unterrichtsphasen mit allen Schüler/innen der Stammklasse Kontakt haben, bauen sie vermehrt auch Beziehungen zu Lernenden außerhalb ihrer Fach- oder Niveaukurse auf. Dies wirkt sich günstig aufs Schulklima aus und hat den Vorteil, dass sie einander bei Konflikten mit Lernenden wirksam entlasten können. ,Ich muss mit einem Schüler nicht kämpfen bis es nicht mehr geht, sondern ich kann kommen und finden, so, jetzt ist fertig [...], kannst du dort mal schauen gehen [...], also, so verhärtete Situationen, [...] die kann man als Team eben wirklich anders tragen“ (LP_E_t1). Die Kooperationskultur, die durch gemeinsame Unterrichtsentwicklung entsteht, wird positiv beurteilt. „Also ich möchte es gar nicht anders haben, als es jetzt ist, ich finde das sehr, sehr angenehm und immer noch äußerst entlastend [...] einfach diese Offenheit, das finde ich absolut befreiend“ (LP_D_t2). Der starke Rückhalt im Kollegium wird besonders bei 
beruflichen und persönlichen Schwierigkeiten sehr geschätzt. „Du findest eigentlich immer jemanden [...], wenn dich gerade etwas beschäftigt“" (LP_D_t3).

\subsubsection{Gemeinsam verantworteter Unterricht (3c)}

Was gemeinsam verantworteten Unterricht anlangt, so werden in den Interviews primär Aspekte der kokonstruktiven Kooperationspraxis beschrieben und beurteilt. Auf Veränderungen im Untersuchungszeitraum hingegen wird kaum eingegangen. Thematisiert wird der Umgang mit Inhalten und Lernzielen. „Die Themen sind die gleichen, die machen wir auch fächerübergreifend ab miteinander, und die Lernziele sind dann differenziert, einfach, mal für den Typ oder für das Niveau“ (LP_E_t1). Beim Teamteaching in offenen Unterrichtsphasen kann es vorkommen, dass z. B. die Deutschlehrerin eine Mathematikaufgabe erklärt oder der Heilpädagoge leistungsstarken Schüler/innen bei Bewerbungsschreiben hilft. „Und für die Kinder [ist es] toll, dass sie sich an unterschiedliche [Personen wenden] können“ (LP_M_t1). Auf Grund der engen Kooperation können die Lehrpersonen niederschwellig Fächer abtauschen. Auch gegenseitige Stellvertretungen sind einfach zu realisieren. „Man kann in die Klassen gehen und es funktioniert einfach“ (LP_D_t1).

\section{Diskussion}

Gesellschaftliche Transformationen in Demografie, Technologien und Arbeitsmarkt und daraus resultierende Bildungsanforderungen haben das Konzept der Personalisierung schulischen Lehrens und Lernens zu einem zunehmend wichtigen bildungspolitischen und pädagogischen Leitmotiv werden lassen. Auch in der Schweiz stellen sich immer mehr Schulen der Aufgabe, fachlich und überfachlich ertragreiche Lernumgebungen, welche die personalen Voraussetzungen der Lernenden besser berücksichtigen, zu gestalten und erweiterte Unterrichtsarchitekturen für eine heterogene Schülerschaft zu entwickeln. Wenn solche Bemühungen Erfolg haben sollen, müssen die Lehrpersonen dieser Schulen über Jahre hinweg intensiv kooperieren. Indem sie als „Communities of Practice“ (CoP) (Wenger 1998) ein schulspezifisches Unterrichtskonzept für personalisiertes Lernen entwickeln und erproben, schul- und unterrichtsrelevante Herausforderungen bei der Umsetzung gemeinsam angehen und den Unterricht kollektiv verantworten, verändert sich auch ihre Kooperation.

Im vorliegenden Beitrag wurde untersucht, inwiefern sich die Kooperation zwischen Lehrpersonen in 43 Schulen, die ihren Unterricht in Richtung personalisierte Lehr-Lernsettings weiterentwickeln, im Zeitraum von drei Schuljahren verändert. Die Ergebnisse der längsschnittlich angelegten Mixed-Methods-Studie zeigen, dass die Lehrpersonen der untersuchten Schulen rege und kokonstruktiv mit Regel- und Speziallehrpersonen kooperieren. Im Untersuchungszeitraum nahm die Häufigkeit der kokonstruktiven Lehrpersonen-Kooperation außer beim gemeinsam verantworteten Unterricht deutlich zu. Die Auswertungen der Fragebogen- und der Interviewdaten aus denselben Schulen führen zu sehr ähnlichen Ergebnissen. Aus den Interviews geht zudem hervor, dass in den als CoP agierenden Lehrpersonenteams eine Kooperationskultur entsteht, die auf Offenheit und Vertrauen beruht, bei beruflichen 
und persönlichen Schwierigkeiten starken Rückhalt gibt, die Unterrichtsentwicklung voranbringt und zu Erträgen führt, die zu erreichen im Alleingang kaum möglich wäre.

Die im Vergleich zum referierten Forschungsstand hoch erscheinende Frequenz kokonstruktiver Lehrpersonen-Kooperation in unserer Studie dürfte einerseits der speziellen Untersuchungsgruppe, anderseits jedoch auch der Komplexität der Entwicklungsaufgabe geschuldet sein. In den perLen-Schulen unterrichten meist erfahrene Lehrpersonen, die langfristig ein gemeinsames Ziel verfolgen, nämlich als CoP die Lehr-Lernkultur in ihrer Schule nachhaltig zu verändern, und die sich eine hohe kollektive Selbstwirksamkeit zuschreiben sowie von unterstützenden Schulleitungen, hoher Arbeitszufriedenheit und geringem Belastungserleben berichten (Florin et al. im Druck). Es handelt sich um reformfreudige Kollegien mit teils visionären Schulleitungen, die Lehrpersonen mit ähnlichen pädagogischen Einstellungen und didaktischen Präferenzen anziehen und ihrer Schule ein spezielles Profil verleihen wollen (Galle im Druck). Dazu kommt, dass Unterrichtsentwicklung in Richtung einer stärkeren Personalisierung des Lernens, anders als bspw. ,Schulische Integration ‘ oder ,Zwei Fremdsprachen in der Primarschule', bottom-up erfolgt bzw. keine verordnete Innovation ist, bei der ein vorgegebenes Konzept lokal angepasst und anhand behördlicher Leitfäden implementiert werden muss. Es ist eine mehrdimensionale und vergleichsweise offene Gestaltungsaufgabe. Jede perLen-Schule entwickelt ein originäres, zur lokalen Situation passendes Personalisierungskonzept und setzt dieses in eigener Regie um. Damit geht ein hoher Aushandlungs- und Kooperationsbedarf einher, was sich in den Ergebnissen unserer Studie widerspiegelt.

Die Häufigkeit der kokonstruktiven Lehrpersonen-Kooperation hat im Untersuchungszeitraum von drei Schuljahren deutlich und bei denselben Gegenständen zugenommen. Besonders ausgeprägt ist der Anstieg der engen Zusammenarbeit bei Problemen mit der Klasse oder der Arbeit. Dies lässt darauf schließen, dass sich bei der mehrjährigen Unterrichtsentwicklung zu personalisiertem Lernen gegenseitiges Vertrauen, Wertschätzung und sozialer Zusammenhalt in den CoP soweit vertieft haben, dass die Lehrpersonen nun nicht nur hart am Unterricht, sondern sogar in persönlich sensiblen Bereichen eng kooperieren. Dass die kokonstruktive Kooperation sich nicht in mehr gemeinsam verantwortetem Unterricht niederschlägt, dürfte an den Rahmenbedingungen liegen. Auch in den perLen-Schulen stehen pro Klasse (nur) eine Vollzeitstelle im Regelschulbereich und ein auf wenige Lektionen beschränktes Kontingent für Speziallehrpersonen bzw. für individualisierende Fördermaßnahmen (u. a. Deutsch als Zweitsprache, schulische Heilpädagogik) zur Verfügung. Gemeinsamer Unterricht ist deshalb fast nur als Teamteaching von Regelund Speziallehrperson realisierbar oder wenn die Schüler/innen einer Stammklasse im offenen Unterricht, der in den perLen-Schulen ca. ein Drittel der Unterrichtszeit beansprucht, von mehreren Lehrpersonen zeitgleich unterstützt werden.

Die Lehrpersonen der perLen-Schulen beurteilen die Kooperation bei der Unterrichtsentwicklung vorwiegend positiv. Dies dürfte neben den Rahmenbedingungen auch mit den wahrgenommenen Veränderungen der kokonstruktiven Kooperation und den damit einher gehenden Wirkungen auf das Lernen der Schüler/innen zusammenhängen (Stebler et al. 2018). Die Kollegien der untersuchten Schulen sind keine fremdbestimmten reformgebeutelten Schicksalsgemeinschaften. Es sind selbst- 
wirksame CoP, die - wenn sie die Anfangshürden der Zusammenarbeit genommen haben - den Mehrwert einer intensiven Kooperation für die Qualität ihres professionellen Handelns erkennen und schätzen und deren Mitglieder ihre beruflichen Kompetenzprofile, die sie als Protagonisten einer personalisierten Lehr-Lernkultur benötigen, schrittweise weiterentwickeln.

Kritisch anzumerken ist, dass die dargestellten Ergebnisse auf Selbstberichten beruhen. Somit kann sich hier das normativ erwünschte Bild, dass kokonstruktive Kooperation zwischen Lehrpersonen ein Markenzeichen guter Schulen und deren Intensivierung ein Qualitätsmerkmal erfolgreicher Entwicklungsarbeit ist, widerspiegeln. Die Tatsache, dass sich einerseits in der Gesamt- und der Teilstichprobe im Untersuchungszeitraum sehr ähnliche Charakteristika in der zunehmenden Häufigkeit und in der wahrgenommenen Qualität auf einzelnen Merkmalsdimensionen zeigen, und dass andererseits bei der Teilstichprobe die Ergebnisse der ausgewerteten Fragebogen- und Interviewdaten gut übereinstimmen, spricht, zumal wir auch versucht haben, das Kooperationsverhalten möglichst realitätsnah abzufragen, für die Validität der Befunde. Dennoch müssen die Ergebnisse an weiteren Schulen, die ebenfalls Unterrichtsentwicklung mit dem Ziel der Ausrichtung des Lehrens und Lernens an den personalen schülerseitigen Voraussetzungen betreiben, dabei aber andere Wege im Umgang mit Individualität und Heterogenität begehen, überprüft werden. Unter diesen Vorbehalten lautet unter vorläufiges Fazit: Indem die Lehrpersonen durch gemeinsame Aushandlung, zyklische (iterative) Anpassung und Reflexion ein pädagogisch durchdachtes Personalisierungskonzept entwickeln und dieses über Jahre hinweg schrittweise umsetzen, verändert sich auch die kokonstruktive Kooperation zwischen den Akteur/innen einschließlich ihrer damit verbundenen professionellen Kompetenzen und trägt dazu bei, dass die Kollegien, wenn man auf die Tiefendimensionen des Personalisierungsdiskurses zurückgreift, auch längerfristig als Lerngemeinschaften unterstützend und bildend wirken. „Ohne“ kokonstruktive „Zusammenarbeit würde das gar nicht gehen“ (SL_L_t1).

Funding Open access funding provided by University of Zurich

Open Access Dieser Artikel wird unter der Creative Commons Namensnennung 4.0 International Lizenz veröffentlicht, welche die Nutzung, Vervielfältigung, Bearbeitung, Verbreitung und Wiedergabe in jeglichem Medium und Format erlaubt, sofern Sie den/die ursprünglichen Autor(en) und die Quelle ordnungsgemäß nennen, einen Link zur Creative Commons Lizenz beifügen und angeben, ob Änderungen vorgenommen wurden.

Die in diesem Artikel enthaltenen Bilder und sonstiges Drittmaterial unterliegen ebenfalls der genannten Creative Commons Lizenz, sofern sich aus der Abbildungslegende nichts anderes ergibt. Sofern das betreffende Material nicht unter der genannten Creative Commons Lizenz steht und die betreffende Handlung nicht nach gesetzlichen Vorschriften erlaubt ist, ist für die oben aufgeführten Weiterverwendungen des Materials die Einwilligung des jeweiligen Rechteinhabers einzuholen.

Weitere Details zur Lizenz entnehmen Sie bitte der Lizenzinformation auf http://creativecommons.org/ licenses/by/4.0/deed.de. 


\section{Literatur}

Basham, J. D., Hall, T.E., Carter, R. A., \& Stahl, W.M. (2016). An operationalized understanding of personalized learning. Journal of Special Education Technology, 31(3), 126-136.

Bastian, J. (2007). Einführung in die Unterrichtsentwicklung. Weinheim: Beltz.

Bennemann, E.-M., \& Schönknecht, G. (2016). Pädagogische Professionalität. Die Einführung der Gemeinschaftsschule - neue Anforderungen an Lehrerprofessionalität. In T. Bohl \& A. Wacker (Hrsg.), Die Einführung der Gemeinschaftsschule in Baden-Württemberg (S. 153-171). Münster: Waxmann.

Bloh, T., \& Bloh, B. (2016). Lehrerkooperation als Community of Practice - Zur Bedeutung kollektivimpliziter Wissensbestände für eine kooperationsbedingte Kompetenzentwicklung. Journal for Educational Research Online, 8(3), 207-230.

Bonsen, M., \& Rolff, H.-G. (2006). Professionelle Lerngemeinschaften von Lehrerinnen und Lehrern. Zeitschrift für Pädagogik, 52(2), 167-184.

Brägger, G., Rolff, H.-G., \& Koch, F. (2021). Kooperative Unterrichtsentwicklung. In G. Brägger \& H.G. Rolff (Hrsg.), Lernen mit digitalen Medien (S. 98-129). Weinheim: Beltz.

Florin, M., Reusser, K., Pauli, C., \& Stebler, R. (eingereicht). Welchen Beitrag leistet die Selbstwirksamkeit von Lehrpersonen bei der Entwicklung einer personalisierten Lehr-Lernkultur? Schweizerische Zeitschrift für Bildungswissenschaften.

Fussangel, K., \& Gräsel, C. (2014). Forschung zur Kooperation im Lehrerberuf. In E. Terhart, H. Bennewitz \& M. Rothland (Hrsg.), Handbuch der Forschung zum Lehrerberuf (2. Aufl. S. 826-864). Münster: Waxmann.

Galle, M. (in Druck). Unterrichtszentrierte Schulentwicklung: Schulen auf den Weg zu einer personalisierten Gestaltung von Lehr-Lernprozessen. Dissertation. Zürich: Universität Zürich.

Gast, I., Schildkamp, K., \& van der Veen, J. T. (2017). Team-based professional development interventions in higher education: a systematic review. Review of Educational Research, 87(4), 736-767.

Gräsel, C., Fussangel, K., \& Pröbstel, C. (2006). Lehrkräfte zur Kooperation anregen - eine Aufgabe für Sisyphos? Zeitschrift für Pädagogik, 52(2), 205-219.

Grosche, M., Fussangel, K., \& Gräsel, C. (2020). Kokonstruktive Kooperation zwischen Lehrkräften. Aktualisierung und Erweiterung der Kokonstruktionstheorie sowie deren Anwendung am Beispiel schulischer Inklusion. Zeitschrift für Pädagogik, 66(4), 461-479.

Gross, B., Tuchman, S., \& Patrick, S. (2018). A national landscape scan of personalized learning in K-12 education in the United States. Vienna: iNACOL.

Haenisch, H., \& Steffens, U. (2017). Schlüsselfaktoren für die Entwicklung von Schulen. In U. Steffens, K. Maag Merki \& H. Fend (Hrsg.), Schulgestaltung. Aktuelle Befunde und Perspektiven der Schulqualitäts- und Schulentwicklungsforschung. Grundlagen der Qualität von Schule 2 (S. 159-184). Münster: Waxmann.

Halbheer, U., Kunz, A., \& Maag Merki, K. (2008). Kooperation zwischen Lehrpersonen in Zürcher Gymnasien: Eine explorative Fallanalyse zum Zusammenhang zwischen kooperativ-reflexiven Prozessen in Schulen und schulischen Qualitätsmerkmalen. Zeitschrift für Soziologie der Erziehung und Sozialisation, 28(1), 19-35.

Harazd, B., \& Drossel, K. (2011). Formen der Lehrerkooperation und ihre schulischen Bedingungen. Empirische Untersuchung zur kollegialen Zusammenarbeit und Schulleitungshandeln. Empirische Pädagogik, 25(2), 145-160.

Hartmann, U., Richter, D., \& Gräsel, C. (2020). Same Same But Different? Analysen zur Struktur kollegialer Kooperation unter Lehrkräften im Kontext von Schul- und Unterrichtsentwicklung. Unterrichtswissenschaft. https://doi.org/10.1007/s42010-020-00090-8.

Keller-Schneider, M., \& Albisser, S. (2013). Kooperation von Lehrpersonen und die Bedeutung von individuellen und kollektiven Ressourcen. In M. Keller-Schneider, S. Albisser \& J. Wissinger (Hrsg.), Professionalität und Kooperation in Schulen. Beiträge zur Diskussion über Schulqualität (S. 33-56). Bad Heilbrunn: Klinkhardt.

Kuckartz, U. (2018). Qualitative Inhaltsanalyse. Methoden, Praxis, Computerunterstützung (4. Aufl.). Weinheim: Beltz.

Kuper, H., \& Kapelle, N. (2012). Lehrerkooperation aus organisationssoziologischer Sicht. In E. Baum, T.-S. Idel \& H. Ullrich (Hrsg.), Kollegialität und Kooperation in der Schule: Theoretische Konzepte und empirische Befunde (S. 41-51). Wiesbaden: VS.

Lee, D., Huh, Y., Lin, C.-Y., Reigeluth, C. M., \& Lee, E. (2021). Differences in personalized learning practice and technology use in high- and low-performing learner-centered schools in the United States. 
Educational Technology Research and Development, 69, 1221-1245. https://doi.org/10.1007/s11423021-09937-y.

Lütje-Klose, B., \& Miller, S. (2017). Kooperation von Lehrkräften mit allgemeinem und sonderpädagogischem Lehramt in inklusiven Settings. Forschungsergebnisse aus Deutschland, Österreich und der Schweiz. In B. Lütje-Klose, S. Miller, S. Schwab \& B. Streese (Hrsg.), Inklusion: Profile für die Schul- und Unterrichtsentwicklung in Deutschland, Österreich und der Schweiz. Theoretische Grundlagen - Empirische Befunde - Praxisbeispiele (S. 203-213). Münster: Waxmann.

Massenkeil, J., \& Rothland, M. (2016). Kollegiale Kooperation im Lehrerberuf. Überblick und Systematisierung aktueller Forschung. Schulpädagogik heute, 7(13), 1-17.

Muckenthaler, M., Tillmann, T., Weiß, S., Hillert, A., \& Kiel, E. (2019). Belastet Kooperation Lehrerinnen und Lehrer? Ein Blick auf unterschiedliche Kooperationsgruppen und deren Belastungserleben. Journal for Educational Research Online, 11(2), 147-168.

Murphy, M., Redding, S., \& Twyman, J. S. (Hrsg.). (2016). Handbook on personalized learning for states, districts, and schools. Philadelphia: Center on Innovations in Learning.

OECD (2014). Talis 2013 results: an international perspective on teaching and learning. Paris: TALIS, OECD Publishing.

Pane, J. F., Steiner, E. D., Baird, M. D., Hamilton, L. S., \& Pane, J. D. (2017). Informing progress: insights on personalized learning. Implementation and effects. Santa Monica: RAND Corporation.

Prain, V., Cox, P., Deed, C., Edwards, D., Farrelly, C., Keeffe, M., et al. (Hrsg.). (2015). Personalising learning in open-plan schools. Rotterdam: Sense.

Pröbstel, C., \& Soltau, A. (2012). Wieso Lehrkräfte (nicht) kooperieren. Die Bedeutung ,,personaler Faktoren“ in der Zusammenarbeit am Arbeitsplatz Schule. In E. Baum, T.-S. Idel \& H. Ullrich (Hrsg.), Kollegialität und Kooperation in der Schule. Theoretische Konzepte und empirische Befunde (S. 55-75). Wiesbaden: Springer.

Reusser, K. (2019). Unterricht als Kulturwerkstatt in bildungswissenschaftlich-psychologischer Sicht. In U. Steffens \& R. Messner (Hrsg.), Unterrichtsqualität. Konzepte und Bilanzen gelingenden Lehrens und Lernens (S. 129-166). Münster: Waxmann.

Reusser, K., Stebler, R., Mandel, D., \& Eckstein, B. (2013). Erfolgreicher Unterricht in heterogenen Lerngruppen auf der Volksschulstufe des Kantons Zürich. Wissenschaftlicher Bericht. Institut für Erziehungswissenschaft: Universität Zürich. https://edudoc.ch/record/108236?ln=de. Zugegriffen: 14.07.2021.

Richter, D., \& Pant, H. A. (2016). Lehrerkooperation in Deutschland. Eine Studie zu kooperativen Arbeitsbeziehungen bei Lehrkräften der Sekundarstufe I. Gütersloh: Bertelsmann Stiftung.

Rolff, H.-G. (2015). Professionelle Lerngemeinschaften als Königsweg. In H.-G. Rolff (Hrsg.), Handbuch Unterrichtsentwicklung (S. 560-571). Weinheim: Beltz.

Schaumburg, H. (2021). Personalisiertes Lernen mit digitalen Medien als Herausforderung für die Schulentwicklung: Ein systematischer Forschungsüberblick. MedienPädagogik: Zeitschrift für Theorie und Praxis der Medienbildung, 41, 134-166.

Schmidt, C. (2013). Auswertungstechniken für Leitfadeninterviews. In B. Friebertshäuser, A. Langer \& A. Prengel (Hrsg.), Handbuch Qualitative Forschungsmethoden in der Erziehungswissenschaft (4. Aufl. S. 473-486). Weinheim: Beltz.

Schratz, M., Schwarz, J.F., \& Westfall-Greiter, T. (2011). Personale Bildungsprozesse in heterogenen Gruppen. Zeitschrift für Bildungsforschung, 1(1), 25-39.

Sebba, J., Brown, N., Steward, S., Galton, M., James, M., Celentano, N., et al. (2007). An investigation of personalised learning approaches used by schools. Brigthon: University of Sussex.

Soltau, A., \& Mienert, M. (2009). Teamorientierung und Einstellungen zu Formen der Lehrerkooperation bei Lehrkräften. Psychologie in Erziehung und Unterricht, 56(3), 213-223.

Spieß, E. (2004). Kooperation und Konflikt. In H. Schuler (Hrsg.), Organisationspsychologie - Gruppe und Organisation. Göttingen: Hogrefe.

Stebler, R., Pauli, C., \& Reusser, K. (2018). Personalisiertes Lernen - Zur Analyse eines Bildungsschlagwortes und erste Ergebnisse aus der perLen-Studie. Zeitschrift für Pädagogik, 64(2), 159-178.

Stebler, R., Pauli, C., \& Reusser, K. (2021a). Personalisiertes Lernen als schulisches Bildungskonzept. Erscheinungsformen, Qualitätsmerkmale und Forschungsbefunde. In G. Brägger \& H.-G. Rolff (Hrsg.), Lernen mit digitalen Medien (S. 400-428). Weinheim: Beltz.

Stebler, R., Pauli, C., \& Reusser, K. (2021b). Personalisiertes Lernen in Schulen der Deutschschweiz. Ergebnisse der perLen-Studie. In G. Brägger \& H.-G. Rolff (Hrsg.), Lernen mit digitalen Medien (S. 429-454). Weinheim: Beltz.

Steinert, B., Klieme, E., Maag Merki, K., Döberich, D., Halbheer, U., \& Kunz, A. (2006). Lehrerkooperation in der Schule: Konzeption, Erfassung, Ergebnisse. Zeitschrift für Pädagogik, 52(2), 185-203. 
Trumpa, S., Franz, E.-K., \& Greiten, S. (2016). Forschungsbefunde zur Kooperation von Lehrkräften. Ein narratives Review. Die Deutsche Schule, 108(1), 80-92.

Tyack, D., \& Tobin, W. (1994). The grammar of schooling: Why has it been so hard to change? American Educational Research Journal, 31, 453-479.

Vangrieken, K., Meredith, C., Packer, T., \& Kyndt, E. (2017). Teacher communities as a context for professional development: a systematic review. Teaching and Teacher Education, 61, 47-59.

Wenger, E. (1998). Communities of practice: learning, meaning, and identity. Cambridge: Cambridge University Press.

Zhang, L., Basham, J.D., \& Yang, S. (2020). Understanding the implementation of personalized learning: a research synthesis. Educational Research Review, 31, 100339. 